\title{
Communication \\ CosR Regulation of perR Transcription for the Control of Oxidative Stress Defense in Campylobacter jejuni
}

\author{
Myungseo Park ${ }^{1,+}$, Sunyoung Hwang $2,3,+, \ddagger$, Sangryeol Ryu $2,3,4, *$ DD and Byeonghwa Jeon $1, *$ (D) \\ 1 Division of Environmental Health Sciences, School of Public Health, University of Minnesota, \\ Minneapolis, MN 55455, USA; park2421@umn.edu \\ 2 Department of Food and Animal Biotechnology, Research Institute for Agriculture and Life Sciences, \\ Seoul National University, Seoul 08826, Korea; skyborn7@korea.kr \\ 3 Department of Agricultural Biotechnology, Research Institute for Agriculture and Life Sciences, \\ Seoul National University, Seoul 08826, Korea \\ 4 Center for Food Bioconvergence, Seoul National University, Seoul 08826, Korea \\ * Correspondence: sangryu@snu.ac.kr (S.R.); bjeon@umn.edu (B.J.) \\ + The authors equally contributed. \\ $\ddagger$ Current address: Food Microbiology Division/Food Safety Evaluation Department, National Institute of \\ Food and Drug Safety Evaluation, Osong 28159, Korea.
}

check for updates

Citation: Park, M.; Hwang, S.; Ryu, S.; Jeon, B. CosR Regulation of perR Transcription for the Control of Oxidative Stress Defense in Campylobacter jejuni. Microorganisms 2021, 9, 1281. https://doi.org/ $10.3390 /$ microorganisms 9061281

Academic Editor: Grzegorz Wegrzyn

Received: 5 May 2021

Accepted: 9 June 2021

Published: 11 June 2021

Publisher's Note: MDPI stays neutral with regard to jurisdictional claims in published maps and institutional affiliations.

Copyright: (c) 2021 by the authors. Licensee MDPI, Basel, Switzerland. This article is an open access article distributed under the terms and conditions of the Creative Commons Attribution (CC BY) license (https:// creativecommons.org/licenses/by/ $4.0 /)$.

\begin{abstract}
Oxidative stress resistance is an important mechanism to sustain the viability of oxygensensitive microaerophilic Campylobacter jejuni. In C. jejuni, gene expression associated with oxidative stress defense is modulated by PerR (peroxide response regulator) and CosR (ampylobacter $\underline{\text { oxidative }}$ stress regulator). Iron also plays an important role in the regulation of oxidative stress, as high iron concentrations reduce the transcription of perR. However, little is known about how iron affects the transcription of $\cos R$. The level of $\cos R$ transcription was increased when the defined media MEM $\alpha$ (Minimum Essential Medium) was supplemented with ferrous $\left(\mathrm{Fe}^{2+}\right)$ and ferric $\left(\mathrm{Fe}^{3+}\right)$ iron and the Mueller-Hinton (MH) media was treated with an iron chelator, indicating that iron upregulates $\cos R$ transcription. However, other divalent cationic ions, such as $\mathrm{Zn}^{2+}, \mathrm{Cu}^{2+}, \mathrm{Co}^{2+}$, and $\mathrm{Mn}^{2+}$, did not affect $\cos R$ transcription, suggesting that $\cos R$ transcription is regulated specifically by iron. Interestingly, the level of perR transcription was increased when CosR was overexpressed. The positive regulation of perR transcription by CosR was observed both in the presence or in the absence of iron. The results of the electrophoretic mobility shift assay showed that CosR directly binds to the perR promoter. DNase I footprinting assays revealed that the CosR binding site in the perR promoter overlaps with the PerR box. In the study, we demonstrated that $\cos R$ transcription is increased in iron-rich conditions, and CosR positively regulates the transcription of PerR, another important regulator of oxidative stress defense in $C$. jejuni. These results provide new insight into how $C$. jejuni regulates oxidative stress defense by coordinating the transcription of per $R$ and $\cos R$ in response to iron.
\end{abstract}

Keywords: Campylobacter; oxidative stress; CosR; PerR

\section{Introduction}

Campylobacter spp. are a leading bacterial cause of gastroenteritis worldwide, accounting for approximately 166 million illnesses and 37,600 deaths per year [1]. Human infections with Campylobacter jejuni, the major pathogenic species of Campylobacter, may develop severe abdominal cramps, watery or bloody diarrhea [2], and, in some cases, induce Guillain-Barré syndrome, an acute flaccid paralysis [3]. Because C. jejuni is a commensal bacterium in the gastrointestinal tract of poultry, human campylobacteriosis is caused most frequently by the consumption of contaminated poultry [4]. C. jejuni requires low oxygen concentrations, such as $3-15 \%$, for growth, but is sensitive to oxygen concentrations in normal aerobic conditions. Thus, aerotolerance plays an important role in the survival of 
C. jejuni during zoonotic transmission from poultry to humans [5-7], and oxidative stress defense is the key mechanism underlying the aerotolerance of $C$. jejuni $[6,8]$.

Usually, genes encoding enzymes involved in the detoxication of reactive oxygen species (ROS) are present redundantly in the genomes of bacteria. However, C. jejuni possesses only a sole copy of genes encoding ROS detoxification enzymes, such as alkyl hydroperoxide reductase ( $\mathrm{AhpC}$ ), catalase (KatA), and superoxide dismutase (SodB). In C. jejuni, the expression of genes of oxidative stress defense is regulated mainly by PerR (peroxide response regulator) and CosR (Campylobacter oxidative stress regulator). OxyR and SoxRS are common regulators of oxidative stress defense in many Gram-negative bacteria [9,10]; however, their orthologs are not present in C. jejuni. In Gram-positive bacteria, PerR is the counterpart of the OxyR of Gram-negative bacteria, which regulates peroxide stress defense. C. jejuni is one of few Gram-negative bacteria that harbor PerR, not OxyR [11]. PerR is a repressor of genes encoding peroxide resistance enzymes (e.g., AhpC and KatA) by directly binding to their promoters [8,11]; thus, the inactivation of per $R$ makes $C$. jejuni hyper-resistant to $\mathrm{H}_{2} \mathrm{O}_{2}$ by derepressing the transcription of ahpC and katA, also increasing aerotolerance in C. jejuni $[8,11]$. Despite the well-known function of PerR in peroxide stress defense, we previously demonstrated that $C$. jejuni PerR regulates the transcription of $\operatorname{sodB}$, the sole gene involved in the detoxification of superoxide resistance [12], showing that PerR regulates both peroxide and superoxide stress defense in C. jejuni.

CosR is another important regulator of oxidative stress defense in C. jejuni. CosR is an OmpR-type response regulator, and its homologs are found predominantly in the bacteria of $\varepsilon$-Proteobacteria, such as Campylobacter, Helicobacter, and Wolinella [13]. In thermotolerant Campylobacter spp., such as C. jejuni, Campylobacter coli, and Campylobacter lari, CosR is an orphan response regulator because no potential sensor kinase is available in the vicinity of $\cos R$; however, the sensor kinase CosS is present in non-thermotolerant Campylobacter spp., such as Campylobacter fetus, Campylobacter concisus, and Campylobacter hominis [14]. Because the function of CosR is essential for the viability of $C$. jejuni, a knockout mutation of $\cos R$ leads to cell death, and its knockout mutant cannot be constructed $[13,15,16]$. Using antisense-mediated gene knockdown, in our previous studies, we identified the regulon of CosR and characterized its function in the regulation of oxidative stress defense [13,17], discovering that $\operatorname{Cos} R$ regulates the expression of a number of genes involved in oxidative stress defense, such as $\operatorname{ahp} C, \operatorname{kat} A$, and $\operatorname{sodB}[13,17]$.

Several studies have reported that iron is a metal cofactor repressing perR in C. jejuni $[11,18,19]$. Increased iron levels derepress PerR-regulated genes of oxidative stress defense, enabling C. jejuni to respond to oxidative stress. Despite the important roles played by CosR in the regulation of oxidative stress defense in C. jejuni, little has been studied about the association of CosR with iron and PerR to modulate the regulation of oxidative stress defense in C. jejuni. Aiming to fill this knowledge gap, in this study, we investigated how iron affects $\cos R$ transcription and how $\operatorname{Cos} R$ is related to perR transcription.

\section{Materials and Methods}

\subsection{Bacterial Strains and Culture Conditions}

C. jejuni NCTC 11168 and its derivatives were grown at $42{ }^{\circ} \mathrm{C}$ in Mueller-Hinton (MH) media (Difco) or MEM $\alpha$ (Minimum Essential Medium; Gibco, Catalog no. 41061) under microaerobic conditions $\left(5 \% \mathrm{O}_{2}, 10 \% \mathrm{CO}_{2}\right.$, and $\left.85 \% \mathrm{~N}_{2}\right)$. MEM $\alpha$ is a defined medium that is commonly used to control iron concentrations in culture media for C. jejuni [20-22]. Iron levels were controlled by growing C. jejuni in MEM $\alpha$ supplemented with iron or by treating MH media with deferoxamine mesylate (DFMS), an iron chelator [23,24]. For the broth culture, an overnight culture on $\mathrm{MH}$ agar was resuspended in $3 \mathrm{~mL}$ of $\mathrm{MH}$ broth or $\mathrm{MEM} \alpha$ to an $\mathrm{OD}_{600}$ of 0.08 , and the bacterial suspension was microaerobically grown with shaking at $200 \mathrm{rpm}$. Kanamycin $\left(50 \mu \mathrm{g} \mathrm{mL} \mathrm{L}^{-1}\right)$ was occasionally added to the culture media to maintain pMW10 [25] and the $\mathrm{P}_{\cos R}::$ lac $Z$ and $\mathrm{P}_{\text {perR }}::$ lac $Z$ promoter fusion constructs. 


\section{2. $\operatorname{Cos} R$ Knockdown and Overexpression}

Since $\cos R$ is essential for the viability of $C$. jejuni $[13,15,16]$, instead of using gene knockout, the intracellular level of CosR was reduced using gene knockdown with antisense peptide nucleic acids (PNA), as described in our previous studies $[13,17]$. Briefly, CosR-specific PNA (CATTTGTTCTATCCTT), which binds reverse complementarily to the leader sequence spanning the ribosomal binding site and the start codon of $\cos R[13,17]$, was commercially synthesized by PNA Bio (Thousand Oaks, CA, USA). To improve cell permeability, the PNA was conjugated to the permeabilization oligonucleotide (KFFKFFKFFK), as reported previously $[13,17]$. Overnight cultures of $C$. jejuni grown on $\mathrm{MH}$ agar plates were resuspended in culture media to an optical density at $600 \mathrm{~nm}\left(\mathrm{OD}_{600}\right)$ of 0.07 , and CosR-specific PNA was added to the suspension to a final concentration of $1.5 \mu \mathrm{M}$ at the beginning of culture. The intracellular level of CosR was increased using a CosR-overexpression strain that was constructed in our previous study by integrating an extra copy of $\cos R$ into the chromosome of $C$. jejuni [17].

\subsection{Construction of a $P_{\cos R}:$ lacZ Promoter Fusion and $\beta$-Galactosidase Assay}

The $\cos R$-lac $Z$ promoter fusion was constructed using pMW10, a promoterless lac $Z$ shuttle vector [25]. The $\cos R$ promoter and its partial coding region were amplified with the primer pairs of CosR_PF_F(XbaI): CCCTTGAAGAGTCTAGAGACTTTGTAAGCTT and CosR_PF_R(XbaI): CAAGCATCTAGACATACGCAGTCTTTTGTAA). The PCR product was cloned into pMW10 after digestion with $\mathrm{XbaI}$, and the final construct was confirmed with sequencing. The constructed plasmid was introduced to C. jejuni NCTC 11168 by conjugation [25]. The perR-lacZ promoter fusion was constructed in our previous study [18]. $\beta$-Galactosidase assays were performed, as described previously, with some modifications $[18,25,26]$. Briefly, $80 \mu \mathrm{L}$ of bacterial culture and $120 \mu \mathrm{L}$ of the $\beta$-galactosidase assay mix, consisting of $60 \mathrm{mM} \mathrm{Na}_{2} \mathrm{HPO}_{4}, 40 \mathrm{mM} \mathrm{NaH}_{2} \mathrm{PO}_{4}, 10 \mathrm{mM} \mathrm{KCl}, 1 \mathrm{mM} \mathrm{MgSO}_{4}, 36 \mathrm{mM}$ $\beta$-mercaptoethanol, $1.1 \mathrm{mg} / \mathrm{mL}$ ONPG, and 6.7\% PopCulture reagent (MilliporeSigma, St. Louis, MI, USA) were mixed and transferred into a 96-well plate to measure $\mathrm{OD}_{420}$ and $\mathrm{OD}_{600}$. After reading the $\mathrm{OD}_{600}$, the plate was incubated at $35^{\circ} \mathrm{C}$ with shaking, and $\mathrm{OD}_{420}$ was measured every $10 \mathrm{~min}$ for $1 \mathrm{~h}$ in a plate reader (Varioskan, ThermoFisher, Waltham, MA, USA). Occasionally, the defined culture medium MEM $\alpha$ was supplemented with different concentrations of $\mathrm{FeSO}_{4}, \mathrm{CoCl}_{2}, \mathrm{CuCl}_{2}, \mathrm{MnCl}_{2}$, and $\mathrm{ZnCl}_{2}$, which were purchased from MilliporeSigma (St. Louis), to examine the effects of metal ions on the transcription of $\cos R$.

\subsection{Electrophoretic Mobility Shift Assay}

An electrophoretic mobility shift assay (EMSA) using recombinant CosR (rCosR) was performed, as described previously [13,17]. Briefly, Escherichia coli BL21 (DE3) carrying plasmid pET15b:: $\cos R$ was grown to an $\mathrm{OD}_{600}$ of approximately 1.0. After induction with $0.5 \mathrm{mM}$ IPTG for $3 \mathrm{~h}$, rCosR was purified under native conditions using $\mathrm{Ni}^{2+}$ affinity chromatography. The DNA fragments containing the promoter region of perR were PCR-amplified with the primer pairs of perR_F: AGACAAATTTATTGAACATGGAAAAACAAG and perR_R: AGAGATTGAAGGGTATTCTTTTTTAATTTC, purified from agarose gel using a gel extraction kit (Qiagen, Hilden, Germany), and labeled with $\left[\gamma^{-}{ }^{32} \mathrm{P}\right]$ ATP (GE Healthcare, Chicago, IL, USA). After elimination of the unincorporated radioisotope with a MicroSpinTMG-25 column (GE Healthcare), the $0.2 \mathrm{nM}$ of ${ }^{32} \mathrm{P}$-labeled DNA probe was incubated with the purified $\mathrm{rCosR}$ protein at different concentrations $(0,0.8,1.6,2.4$, and $3.2 \mathrm{nM}$ ) at $37{ }^{\circ} \mathrm{C}$ for $15 \mathrm{~min}$ in $10 \mu \mathrm{L}$ of the gel-shift assay buffer ( $20 \mathrm{mM}$ HEPES (pH7.6), $1 \mathrm{mM}$ EDTA, $10 \mathrm{mM}\left(\mathrm{NH}_{4}\right)_{2} \mathrm{SO}_{4}, 5 \mathrm{mM}$ DTT, $0.2 \%$ Tween $20,30 \mathrm{mM} \mathrm{KCl}, 0.1 \mu \mathrm{g}$ poly (dI-dC)). Unlabeled PCR amplicon of the perR promoter, which was prepared with the same method as above without $\left[\gamma^{-}{ }^{32} \mathrm{P}\right]$ ATP, was used as a competitor. The reaction mixtures were resolved in a $6 \%$ polyacrylamide gel, and the radiolabeled DNA fragments were visualized using the BAS2500 system (Fuji Film, Kyoto, Japan). 


\subsection{DNase I Footprinting Assay}

A DNase I footprinting assay was performed following a method described previously $[13,18]$. DNA fragments containing the perR promoter region were PCR amplified using a ${ }^{32}$ P-labeled primer perR_FP_F: AGCCTTGCAAGAAATGAATAATAATGC and an unlabeled primer perR_FP_R: ATTCATCAATATTAGGATGCTCATGTC, and were purified from the agarose gel with Wizard SV Gel and the PCR Clean-Up System (Promega). Binding of rCosR to the ${ }^{32} \mathrm{P}$-labeled perR promoter was performed at $37^{\circ} \mathrm{C}$ for $10 \mathrm{~min}$ in $40 \mu \mathrm{L}$ of the gel-shift assay buffer (20 mM HEPES (pH7.6), $1 \mathrm{mM}$ EDTA, $10 \mathrm{mM}\left(\mathrm{NH}_{4}\right)_{2} \mathrm{SO}_{4}, 5 \mathrm{mM}$ DTT, $0.2 \%$ Tween $20,30 \mathrm{mM} \mathrm{KCl}, 0.1 \mu \mathrm{g}$ poly (dI-dC)) containing $10 \mathrm{mM}$ of $\mathrm{MgCl}_{2}$. After treatment of the reaction mixture with or without $0.1 \mathrm{U}$ DNase I (Takara), the reactions were stopped by the addition of $200 \mu \mathrm{L}$ of ice-cold stop solution (0.4 M NaOAc, $2.5 \mathrm{mM}$ EDTA), and the DNA products were purified by phenol extraction and ethanol precipitation. The digested DNA fragments were separated by electrophoresis in $6 \%$ polyacrylamide- $8 \mathrm{M}$ urea gels alongside sequencing ladders that were generated with the same ${ }^{32} \mathrm{P}$-labeled primer used to amplify DNA fragments for DNase I digestion.

\section{Results}

\subsection{Regulation of $\cos R$ Transcription by Iron}

The level of $\cos R$ transcription was measured with $\beta$-galactosidase assays by supplementing the defined medium MEM $\alpha$ with different concentrations of iron. To control iron concentrations in media, we used MEM $\alpha$, a defined media that have been frequently used to analyze the effects of iron and other metal ions, such as Zn, on the physiology and pathogenicity of $C$. jejuni in many studies $[20-22,27]$ The results of the $P_{\cos R}$-lacZ fusion assay demonstrated that iron increased the level of $\cos R$ transcription in a concentrationdependent manner (Figure 1). The level of $\cos R$ transcription was increased by $27.5 \%$ and $28 \%$ at $2 \mu \mathrm{M} \mathrm{Fe}^{3+}$ and $2 \mu \mathrm{M} \mathrm{Fe}^{2+}$, respectively, compared to the control without iron (Figure 1), suggesting that both $\mathrm{Fe}^{3+}$ and $\mathrm{Fe}^{2+}$ affect $\cos R$ transcription. The assay was also conducted with different divalent cationic ions, such as $\mathrm{Zn}^{2+}, \mathrm{Cu}^{2+}, \mathrm{Co}^{2+}$, and $\mathrm{Mn}^{2+}$, to examine whether $\cos R$ transcription was influenced by other metal ions. However, the transcriptional level of $\cos R$ was not changed by the divalent cationic ions (Figure 2), suggesting that $\cos R$ transcription is regulated specifically by iron.
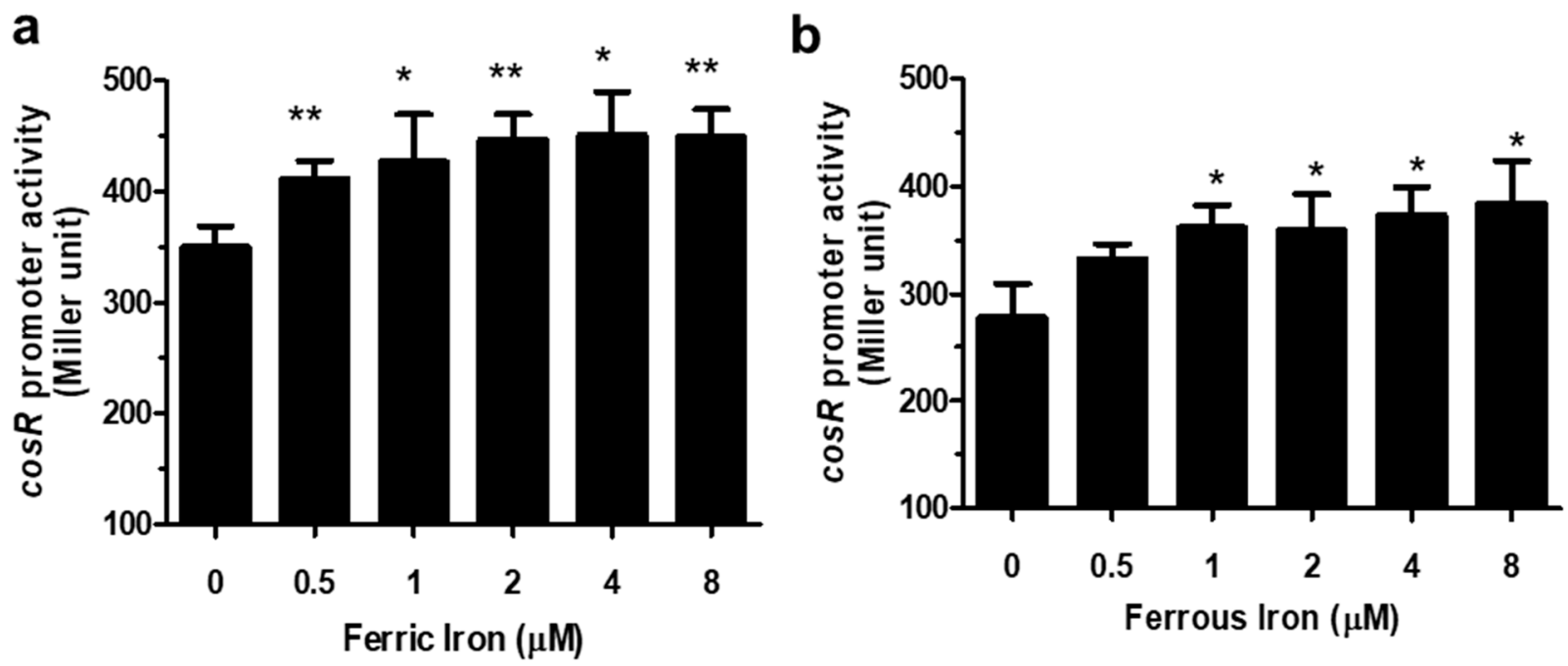

Figure 1. Positive regulation of $\cos R$ transcription by iron. The $\mathrm{P}_{\cos R}:$ :lacZ fusion assay was conducted by supplementing MEM $\alpha$ with different concentrations of $\mathrm{Fe}^{3+}(\mathbf{a})$ and $\mathrm{Fe}^{2+}(\mathbf{b})$. The results show the means and standard deviations of three samples in a single experiment. The experiment was repeated three times, and similar results were obtained in the repeated experiments. The statistical analysis was performed using Student's t-test in comparison with the control without iron. $*: p<0.05,{ }^{* *}: p<0.01$. 
a
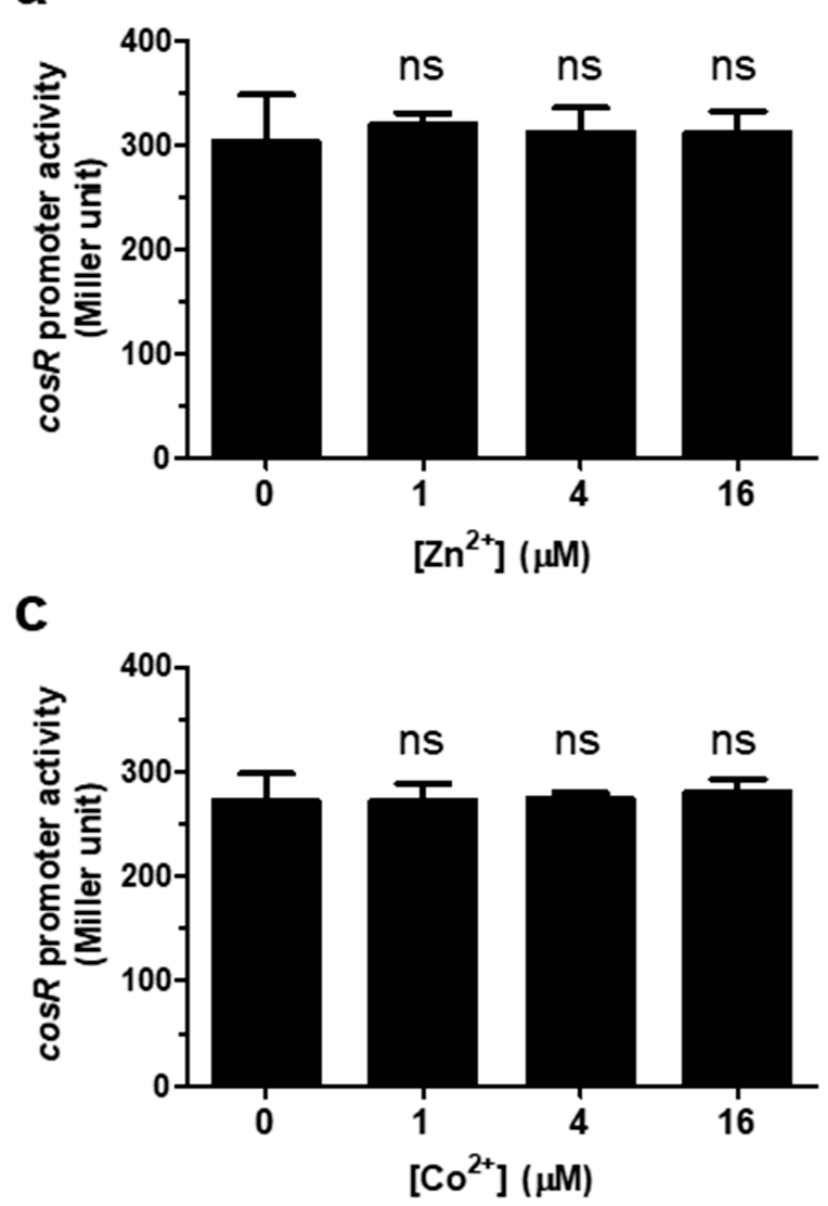

b
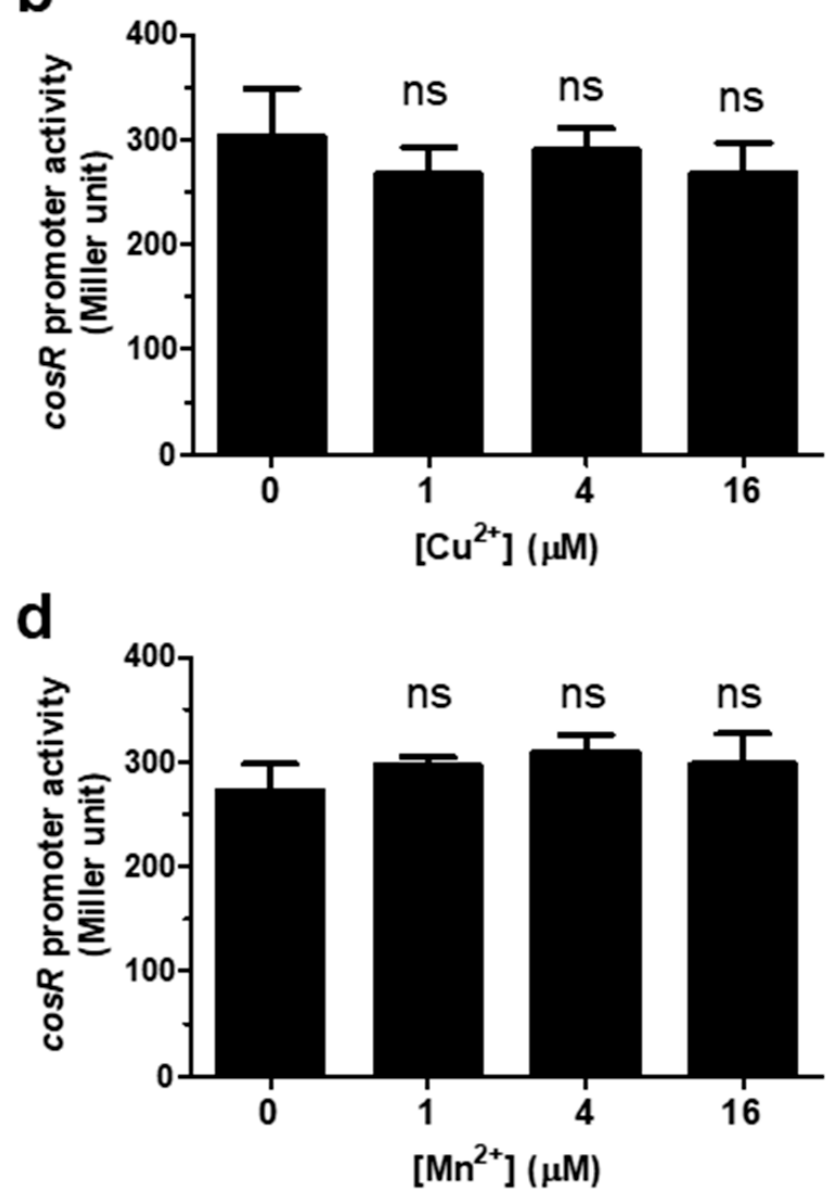

Figure 2. Irresponsiveness of $\cos R$ transcription to divalent metal ions other than iron. The $\mathrm{P}_{\cos R}:$ :lac $Z$ fusion assay was conducted with $\mathrm{Zn}^{2+}(\mathbf{a}), \mathrm{Cu}^{2+}(\mathbf{b}), \mathrm{Co}^{2+}(\mathbf{c})$, and $\mathrm{Mn}^{2+}(\mathbf{d})$. The results show the means and standard deviations of three samples in a single experiment. The experiment was repeated three times, and similar results were obtained in the repeated experiments. The statistical analysis was performed using Student's $t$-test in comparison with the control without ions. ns: not significant.

\subsection{Iron Regulation of $\cos R$ and perR Transcription over the Growth of C. jejuni}

The levels of perR and $\cos R$ transcription were measured in the presence and absence of iron over the growth of $C$. jejuni. Iron levels were controlled by supplementing MEM $\alpha$ with $\mathrm{Fe}^{3+}$ or treating the $\mathrm{MH}$ media with an iron chelator. Regardless of iron, the transcriptional levels of both per $R$ and $\cos R$ were reduced in the lag phase for the first few hours of culture $\operatorname{MEM} \alpha$, and increased in the exponential phase (Figure $3 a, b)$. Because the effect of iron on per $R$ transcription has been reported in several studies $[11,18,19], \mathrm{P}_{\text {per } R}:$ :lac $Z$ was included as a control to compare the expression patterns with $\cos R$. The increase in the level of $\cos R$ transcription by iron was significant both in the exponential and in the stationary phases in MEM $\alpha$ (Figure 3a), and similar patterns were observed when the experiment was performed in $\mathrm{MH}$ media using an iron chelator (Figure 3c). After cultivation in iron-rich conditions for $8 \mathrm{~h}$, the levels of $\cos R$ transcription were increased by $21.1 \%$ and $40.4 \%$ in $\mathrm{MEM} \alpha$ and $\mathrm{MH}$ media, respectively (Figure 3a,c), whereas perR transcription was reduced by $22.2 \%$ and $47.9 \%$ in MEM $\alpha$ and MH media, respectively (Figure $3 b, d$ ). 
a
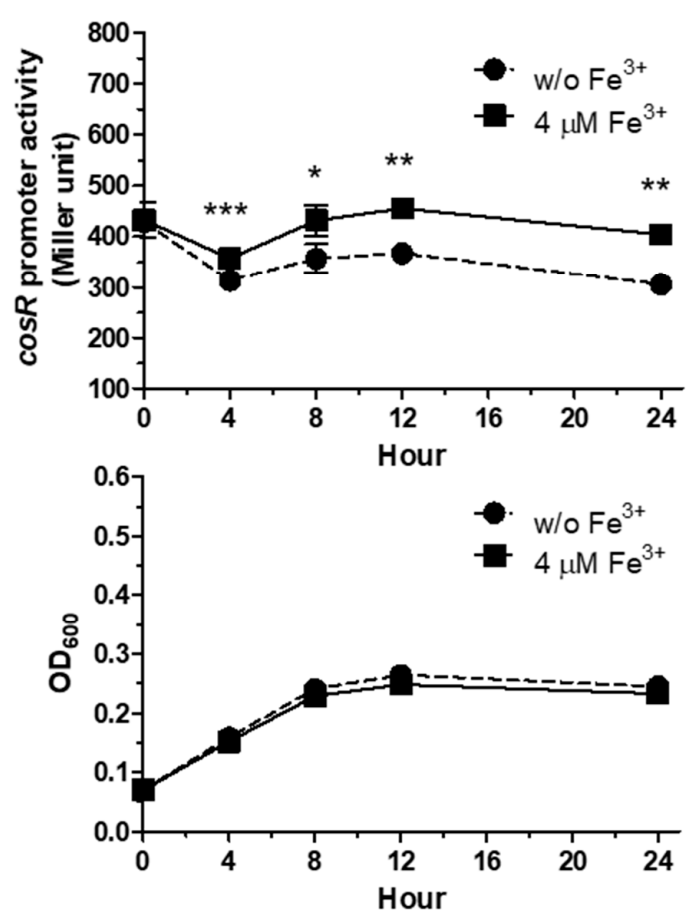

C
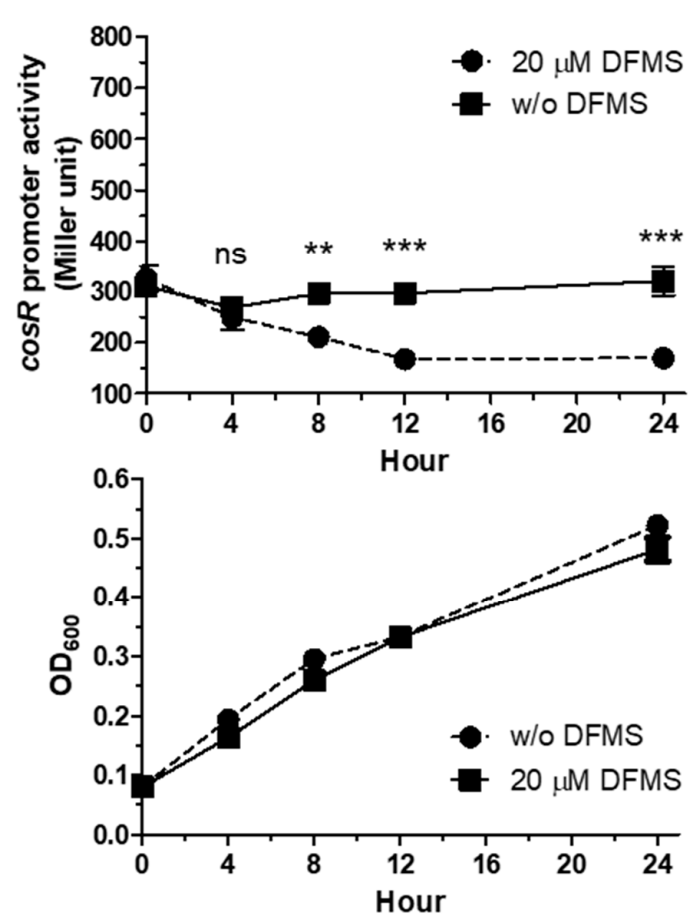

b
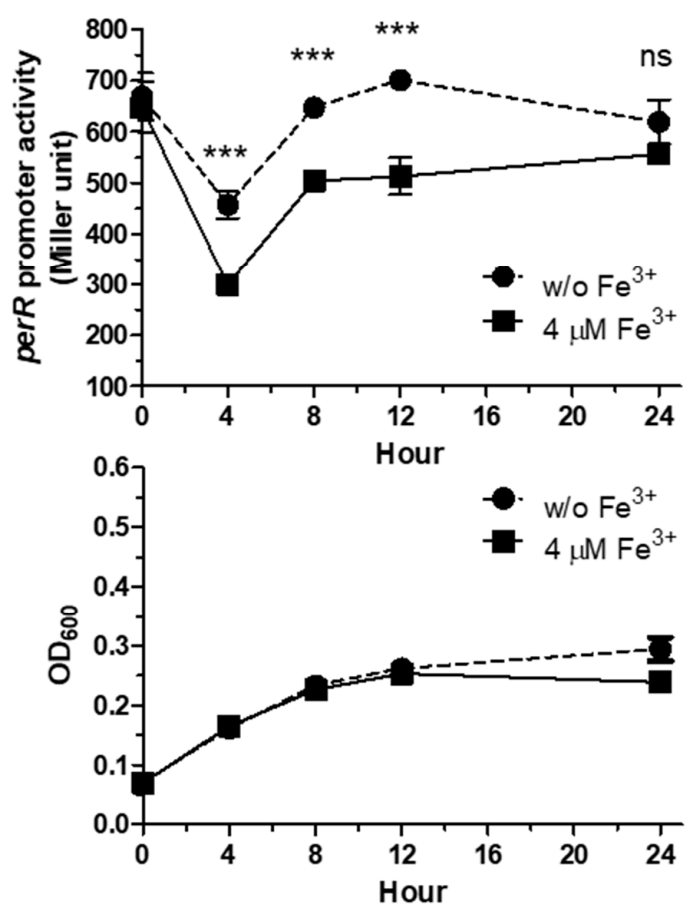

d
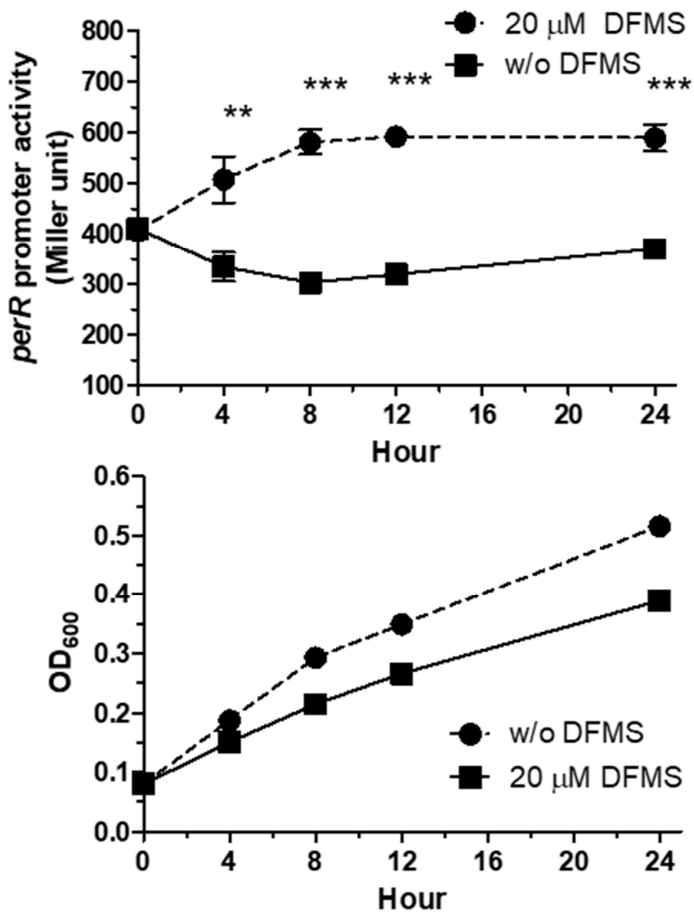

Figure 3. Effects of iron on the transcription of $\cos R$ and perR over the growth of $C$. jejuni. The $\mathrm{P}_{\cos R}:$ :lacZ and $\mathrm{P}_{\text {perR }}::$ lac $Z$ fusion assays were conducted in the presence and absence of iron in MEM $\alpha$ and MH media: (a) $\mathrm{P}_{\cos R}:: l a c Z$ assay in MEM $\alpha$.

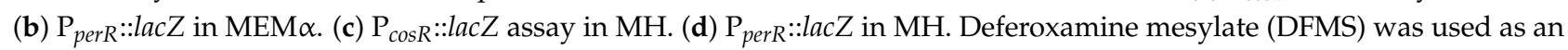
iron chelator in $\mathrm{MH}$ media. The growth of $C$. jejuni is indicated with the optical density at $600 \mathrm{~nm}\left(\mathrm{OD}_{600}\right)$ in each panel. The experiment was repeated three times, and similar patterns of results were obtained in the experiments. The statistical analysis was performed using Student's $t$-test by comparing the samples with and without iron at the same sampling point. ${ }^{*}: p<0.05,{ }^{* *}: p<0.01,{ }^{* * *}: p<0.001$. 


\section{3. $\operatorname{Cos} R$ Regulation of perR Transcription}

Based on the role of CosR and PerR in the regulation of oxidative stress defense, we hypothesized that the two regulators may influence their transcription to coordinate the transcriptional regulation of oxidative stress defense. To examine this hypothesis, we measured whether CosR might affect perR transcription. Due to the essentiality of CosR in the viability of $C$. jejuni, a knockout mutant of $\cos R$ could not be constructed in multiple studies $[13,15,16]$. Thus, we measured the level of perR transcription under the conditions of CosR overexpression and CosR knockdown which were established in our previous studies $[13,17]$. Interestingly, the results of the $\mathrm{P}_{\text {perR }}$-lac $Z$ fusion assay showed that the level of perR transcription was increased when CosR was overexpressed (Figure 4). However, CosR knockdown by antisense PNA did not affect perR transcription significantly (Figure 4). Although the level of perR transcription was increased by CosR overexpression in the presence and absence of iron, the overall level of perR transcription was reduced by iron regardless of the levels of CosR in wild-type, a CosR-overexpression strain, and CosRknockdown conditions (Figure 4), suggesting that CosR regulation of perR transcription is independent of iron.

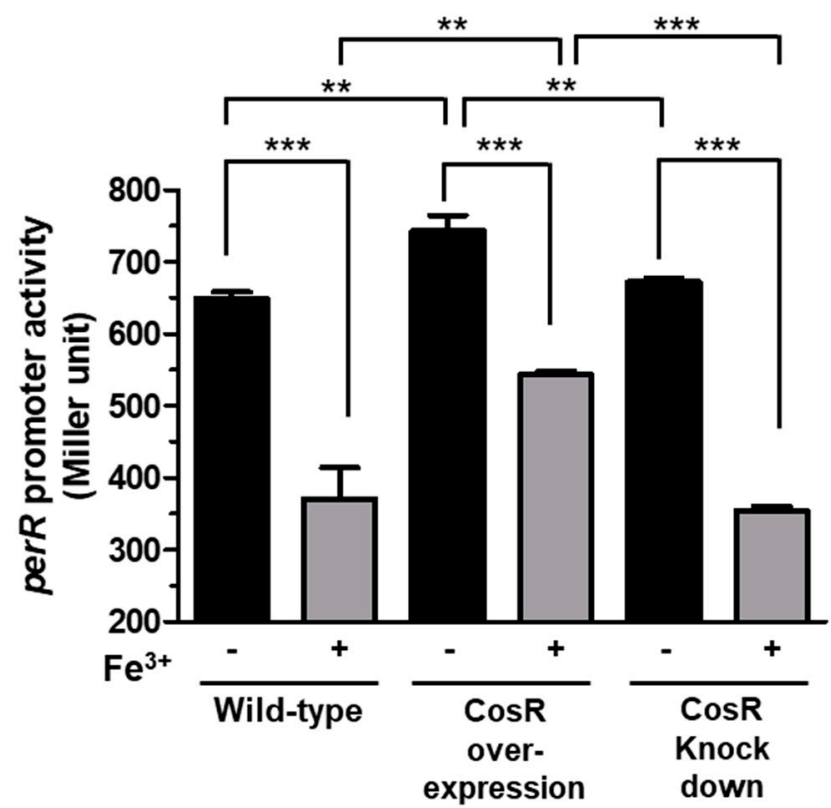

Figure 4. Positive regulation of perR transcription by CosR. The $\mathrm{P}_{\text {perR }}:$ :lac $Z$ fusion assays were conducted under conditions with different levels of CosR, including wild-type, CosR overexpression, and CosR knockdown using antisense PNA. The experiment was repeated three times, and similar results were obtained in the experiments. The statistical analysis was performed using Student's t-test. ${ }^{* *}: p<0.01,{ }^{* * *}: p<0.001$.

\subsection{CosR Binding to the perR Promoter}

Since CosR positively affected perR transcription (Figure 4), we examined whether CosR regulation of perR transcription is mediated by direct interaction between CosR and the perR promoter. The binding of CosR to the perR promoter was examined with EMSA, which showed that CosR was directly bound to the perR promoter (Figure 5a). Previously, we reported that the transcription of perR is driven by two promoters, and PerR regulates its transcription by autoregulation [18]. By performing a DNase I footprinting assay, we identified the CosR binding site in the -10 and -35 region of the two perR promoters (Figure $5 b$ ), and a part of the $5^{\prime}$ region of the CosR binding site that overlapped with the PerR box to which PerR binds for autoregulation (Figure 5c). These results indicate that CosR regulates per $R$ transcription by directly binding to the per $R$ promoter. 
a

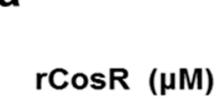
Competitor
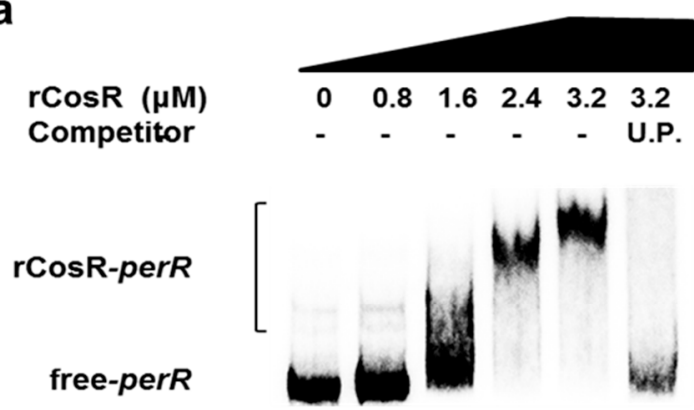

C
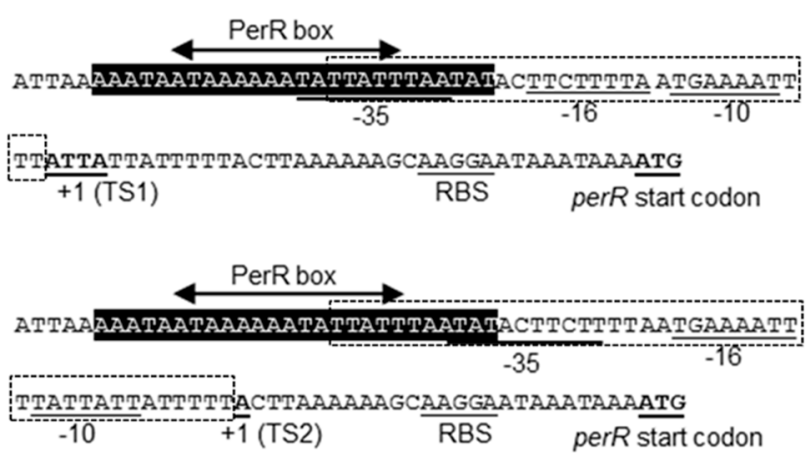

b

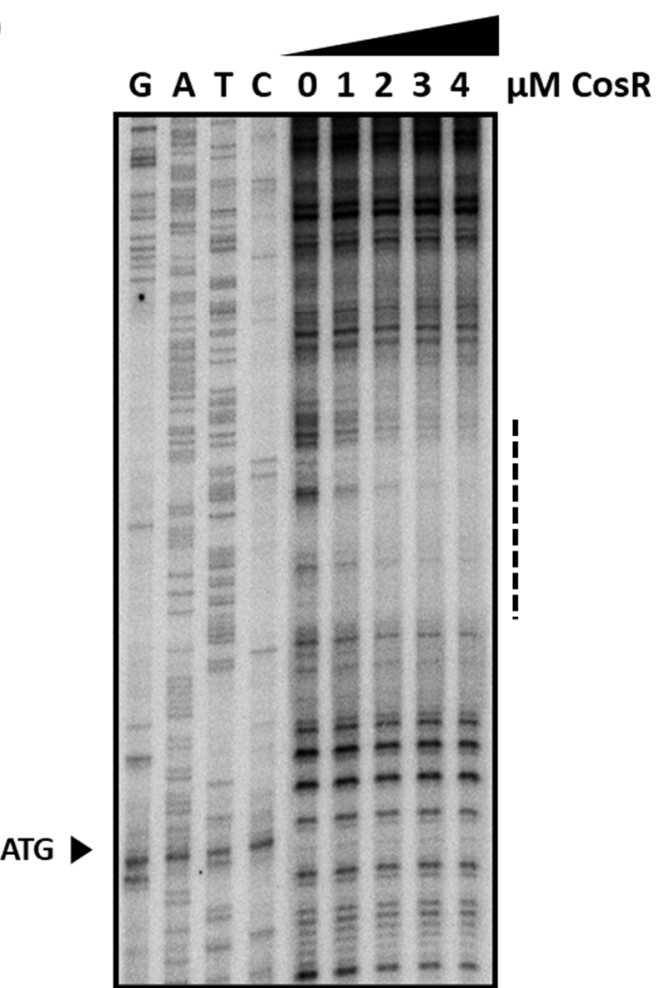

Figure 5. CosR binding to the perR promoter (a) The results of EMSA showed that rCosR bound to the perR promoter. Unlabeled probe (U.P.) is the PCR amplicon of the perR promoter, which was prepared without $[\gamma-32 \mathrm{P}]$ ATP and was used as a competitor. (b) Identification of the CosR binding sites in the perR promoter by DNase I footprinting. The CosR binding region is indicated with a dotted line, and ATC is the start codon of perR. (c) The CosR binding site in the two adjacently located perR promoters, which were reported in a previous study [18]. The PerR box is the site for PerR binding for autoregulation [18]. The perR start codon, the transcriptional start site (+1), and the $-10,-16$, and -35 elements are underlined.

\section{Discussion}

Since C. jejuni is a microaerophilic bacterium sensitive to oxygen levels in normal atmospheric conditions, oxidative stress defense is important for the survival of $C$. jejuni during foodborne transmission to humans through various routes that generally involve aerobic environments. To achieve a timely response to oxidative stress, oxidative stress defense should be coordinated efficiently in which CosR, PerR, and iron play key roles. Iron is an essential micronutrient affecting bacterial growth [28], and the unavailability of iron significantly reduces the growth of $C$. jejuni [22,24]. Moreover, iron participates in the generation of ROS through the Fenton reaction and is also involved in the regulation of oxidative stress defense [22,24], primarily by repressing the transcription of perR in C. jejuni $[11,18]$. PerR is a metal-responsive repressor protein [11,29]. Whereas Bacillus subtilis PerR uses either manganese or iron as a regulatory metal cofactor to detect oxidative stress [30,31], the transcription of perR in C. jejuni is affected only by iron, not manganese [18]. Similarly, the level of $\cos R$ transcription was increased by iron (Figure 1); however, other divalent cationic ions, such as $\mathrm{Zn}^{2+}, \mathrm{Cu}^{2+}, \mathrm{Co}^{2+}$, and $\mathrm{Mn}^{2+}$, did not affect $\cos R$ transcription (Figure 2).

Interestingly, iron affects the transcription of perR and $\cos R$ in the opposite pattern; iron-rich conditions reduce per $R$ transcription but increase $\cos R$ transcription. The opposite patterns in the transcriptional regulation of $\operatorname{per} R$ and $\cos R$ by iron may facilitate the timely response of $C$. jejuni to oxidative stress defense because PerR is a repressor of ROS detoxification enzymes, such as $\mathrm{AhpC}$, KatA, and SodB, whereas CosR is a positive regulator of AhpC and KatA [13,17]. Increased iron levels, which can be accompanied by an increase in oxidative stress, reduce the level of perR transcription, resulting in the depression of $a h p C$, kat $A$, and $\operatorname{sodB}$. (Figure 6). Under iron-rich conditions, however, $\cos R$ transcription 
was increased (Figures 1 and 2), which may consequently increase the levels of AhpC and KatA due to the positive regulation by CosR (Figure 6). In response to increased iron levels, collectively, C. jejuni can stimulate the transcription of the genes encoding ROS detoxification enzymes through the negative regulation of per $R$ transcription and the positive regulation of $\cos R$ transcription, leading to the alleviation of oxidative stress (Figure 6).

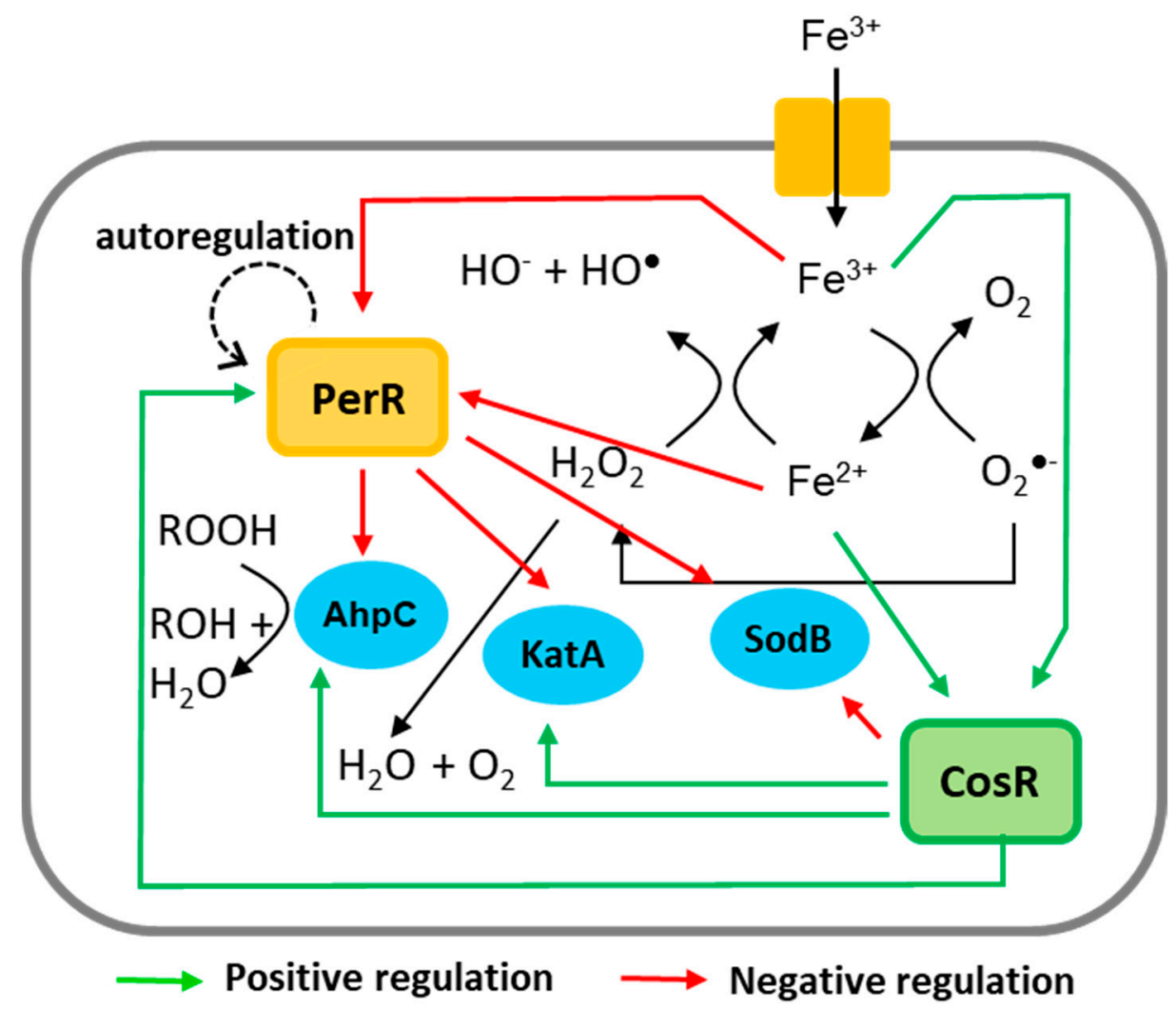

Figure 6. Schematic diagram of oxidative stress defense regulation by CosR and PerR. Positive and negative regulations are indicated with green and red lines, respectively.

In this study, we discovered that CosR positively regulates perR transcription by directly binding to the perR promoter (Figures 4 and 5). CosR regulated perR transcription only when the level of CosR was increased because CosR knockdown using antisense PNA did not affect perR transcription (Figure 4). Although perR transcription was positively regulated by CosR without iron, the level of perR transcription was increased by CosR overexpression more significantly in the presence of iron than the absence of iron (46.5\% increase with iron vs. $14.5 \%$ increase without iron) (Figure 4 ), which can be ascribed to the positive regulation of $\cos R$ transcription by iron. High iron concentrations enhance the expression of ROS detoxification enzymes by repressing perR transcription and increasing $\cos R$ transcription. However, a regulatory mechanism is needed to coordinate the transcriptional levels of perR and $\cos R$ to avoid potential over-stimulation of ROS detoxification systems where CosR regulation of per $R$ transcription may play a role.

The transcription of per $R$ is driven by two adjacently located promoters, both of which are repressed by iron and subjected to autoregulation [18]. The CosR binding region that was identified using a DNase I footprinting assay overlapped with the PerR box to which PerR binds for autoregulation (Figure $5 c$ ). This suggests that PerR and CosR may compete for binding to the perR promoter under certain circumstances.

In the gastrointestinal tract, relatively high concentrations (ca. $25 \mathrm{mM}$ ) of iron are present theoretically; however, only a small proportion of iron is bioavailable because of the low water solubility of inorganic iron [32]. The acquisition of iron affects C. jejuni colonization of the gastrointestinal tracts since the knockout mutation of genes involved in 
iron acquisition results in colonization defects [33-35]. Because oxidative stress defense is a complicated process involving a number of factors, such as the presence of oxygen, iron, and antioxidants, it is difficult to speculate how the findings in this study can impact the regulation of oxidative stress defense, particularly during the colonization of the gastrointestinal tract, where oxygen levels are extremely low. Presumably, the regulation of oxidative stress defense involving CosR, PerR, and iron may contribute to the survival of this microaerophile when exposed to aerobic environments. In our previous study, we demonstrated that iron stimulates biofilm formation in C. jejuni by increasing oxidative stress, suggesting that iron utilization in combination with oxidative stress contributes to the survival of C. jejuni in aerobic environments [20]. At this stage, future studies are still needed to elucidate how oxidative stress defense is regulated to improve the survival of this fastidious bacterium under stress conditions during transmission and infection.

Author Contributions: Conceptualization, S.H., S.R. and B.J.; methodology, M.P., S.H., S.R. and B.J.; validation, S.R. and B.J.; formal analysis, M.P., S.H., S.R. and B.J.; investigation, M.P., S.H., S.R. and B.J.; resources, S.R. and B.J.; writing-original draft preparation, M.P., S.H. and B.J.; writing-review and editing, S.R. and B.J.; visualization, J M.P. and S.H.; supervision, S.R. and B.J.; project administration, S.R. and B.J.; funding acquisition, S.R. and B.J. All authors have read and agreed to the published version of the manuscript. Please turn to the CRediT taxonomy for the term explanation. Authorship must be limited to those who have contributed substantially to the work reported.

Funding: B.J. was supported by MnDRIVE (Minnesota's Discovery, Research, and InnoVation Economy).

Conflicts of Interest: The authors declare no conflict of interest.

\section{References}

1. Kirk, M.D.; Pires, S.M.; Black, R.E.; Caipo, M.; Crump, J.A.; Devleesschauwer, B.; Dopfer, D.; Fazil, A.; Fischer-Walker, C.L.; Hald, T.; et al. World health organization estimates of the global and regional disease burden of 22 foodborne bacterial, protozoal, and viral diseases, 2010: A data synthesis. PLoS Med. 2015, 12, e1001921.

2. Heimesaat, M.M.; Backert, S.; Alter, T.; Bereswill, S. Human campylobacteriosis-a serious infectious threat in a one health perspective. Curr. Top. Microbiol. Immunol. 2021, 431, 1-23. [CrossRef] [PubMed]

3. Wachira, V.K.; Peixoto, H.M.; de Oliveira, M.R.F. Systematic review of factors associated with the development of Guillain-Barre syndrome 2007-2017: What has changed? Trop. Med. Int. Health TM IH 2019, 24, 132-142. [CrossRef] [PubMed]

4. Skarp, C.P.A.; Hänninen, M.L.; Rautelin, H.I.K. Campylobacteriosis: The role of poultry meat. Clin. Microbiol. Infect. 2016, 22, 103-109. [CrossRef]

5. Oh, E.; Chui, L.; Bae, J.; Li, V.; Ma, A.; Mutschall, S.K.; Taboada, E.N.; McMullen, L.M.; Jeon, B. Frequent implication of multistress-tolerant Campylobacter jejuni in human infections. Emerg. Infect. Dis. 2018, 24, 1037-1044. [CrossRef] [PubMed]

6. Oh, E.; McMullen, L.; Jeon, B. Impact of oxidative stress defense on bacterial survival and morphological change in Campylobacter jejuni under aerobic conditions. Front. Microbiol. 2015, 6, 295. [CrossRef] [PubMed]

7. Oh, E.; McMullen, L.M.; Chui, L.; Jeon, B. Differential survival of hyper-aerotolerant Campylobacter jejuni under different gas conditions. Front. Microbiol. 2017, 8, 954. [CrossRef]

8. Handley, R.A.; Mulholland, F.; Reuter, M.; Ramachandran, V.K.; Musk, H.; Clissold, L.; Le Brun, N.E.; van Vliet, A.H.M. PerR controls oxidative stress defence and aerotolerance but not motility-associated phenotypes of Campylobacter jejuni. Microbiology 2015, 161, 1524-1536. [CrossRef]

9. Chiang, S.M.; Schellhorn, H.E. Regulators of oxidative stress response genes in Escherichia coli and their functional conservation in bacteria. Arch. Biochem. Biophys. 2012, 525, 161-169. [CrossRef] [PubMed]

10. Imlay, J.A. Cellular defenses against superoxide and hydrogen peroxide. Annu. Rev. Biochem. 2008, 77, 755-776. [CrossRef] [PubMed]

11. van Vliet, A.H.; Baillon, M.L.; Penn, C.W.; Ketley, J.M. Campylobacter jejuni contains two fur homologs: Characterization of iron-responsive regulation of peroxide stress defense genes by the PerR repressor. J. Bacteriol. 1999, 181, 6371-6376. [CrossRef] [PubMed]

12. Kim, J.C.; Oh, E.; Hwang, S.; Ryu, S.; Jeon, B. Non-selective regulation of peroxide and superoxide resistance genes by PerR in Campylobacter jejuni. Front. Microbiol. 2015, 6, 126. [CrossRef]

13. Hwang, S.; Kim, M.; Ryu, S.; Jeon, B. Regulation of oxidative stress response by CosR, an essential response regulator in Campylobacter jejuni. PLoS ONE 2011, 6, e22300. [CrossRef] [PubMed]

14. Hwang, S.; Miller, W.G.; Ryu, S.; Jeon, B. Divergent distribution of the sensor kinase CosS in non-thermotolerant campylobacter species and its functional incompatibility with the response regulator CosR of Campylobacter jejuni. PLoS ONE 2014, 9, e89774. [CrossRef] 
15. Garénaux, A.; Guillou, S.; Ermel, G.; Wren, B.; Federighi, M.; Ritz, M. Role of the Cj1371 periplasmic protein and the Cj0355c two-component regulator in the Campylobacter jejuni NCTC 11168 response to oxidative stress caused by paraquat. Res. Microbiol. 2008, 159, 718-726. [CrossRef] [PubMed]

16. Raphael, B.H.; Pereira, S.; Flom, G.A.; Zhang, Q.; Ketley, J.M.; Konkel, M.E. The Campylobacter jejuni response regulator, CbrR, modulates sodium deoxycholate resistance and chicken colonization. J. Bacteriol. 2005, 187, 3662-3670. [CrossRef] [PubMed]

17. Hwang, S.; Zhang, Q.; Ryu, S.; Jeon, B. Transcriptional regulation of the CmeABC multidrug efflux pump and the KatA catalase by CosR in Campylobacter jejuni. J. Bacteriol. 2012, 194, 6883-6891. [CrossRef]

18. Kim, M.; Hwang, S.; Ryu, S.; Jeon, B. Regulation of perR expression by iron and PerR in Campylobacter jejuni. J. Bacteriol. 2011, 193, 6171-6178. [CrossRef]

19. Butcher, J.; Handley, R.A.; van Vliet, A.H.; Stintzi, A. Refined analysis of the Campylobacter jejuni iron-dependent/independent Fur- and PerR-transcriptomes. BMC Genom. 2015, 16, 498. [CrossRef]

20. Oh, E.; Andrews, K.J.; Jeon, B. Enhanced biofilm formation by ferrous and ferric iron through oxidative stress in Campylobacter jejuni. Front. Microbiol. 2018, 9, 1204. [CrossRef]

21. Wang, H.; Zeng, X.; Lin, J. Enterobactin-specific antibodies inhibit in vitro growth of different gram-negative bacterial pathogens. Vaccine 2020, 38, 7764-7773. [CrossRef]

22. Palyada, K.; Threadgill, D.; Stintzi, A. Iron acquisition and regulation in Campylobacter jejuni. J. Bacteriol. 2004, 186, 4714-4729. [CrossRef]

23. van Vliet, A.H.; Wooldridge, K.G.; Ketley, J.M. Iron-responsive gene regulation in a Campylobacter jejuni fur mutant. J. Bacteriol. 1998, 180, 5291-5298. [CrossRef]

24. Holmes, K.; Mulholland, F.; Pearson, B.M.; Pin, C.; McNicholl-Kennedy, J.; Ketley, J.M.; Wells, J.M. Campylobacter jejuni gene expression in response to iron limitation and the role of Fur. Microbiology 2005, 151, 243-257. [CrossRef]

25. Wösten, M.M.; Boeve, M.; Koot, M.G.; van Nuenen, A.C.; van der Zeijst, B.A. Identification of Campylobacter jejuni promoter sequences. J. Bacteriol. 1998, 180, 594-599. [CrossRef]

26. Schaefer, J.; Jovanovic, G.; Kotta-Loizou, I.; Buck, M. Single-step method for $\beta$-galactosidase assays in Escherichia coli using a 96-well microplate reader. Anal. Biochem. 2016, 503, 56-57. [CrossRef] [PubMed]

27. Barnawi, H.; Masri, N.; Hussain, N.; Al-Lawati, B.; Mayasari, E.; Gulbicka, A.; Jervis, A.J.; Huang, M.H.; Cavet, J.S.; Linton, D. RNA-based thermoregulation of a Campylobacter jejuni zinc resistance determinant. PLoS Pathog. 2020, 16, e1009008. [CrossRef]

28. Bradley, J.M.; Svistunenko, D.A.; Wilson, M.T.; Hemmings, A.M.; Moore, G.R.; Le Brun, N.E. Bacterial iron detoxification at the molecular level. J. Biol. Chem. 2020, 295, 17602-17623. [CrossRef] [PubMed]

29. Lee, J.W.; Helmann, J.D. Functional specialization within the Fur family of metalloregulators. Biometals 2007, 20, 485-499. [CrossRef] [PubMed]

30. Lee, J.W.; Helmann, J.D. The PerR transcription factor senses $\mathrm{H}_{2} \mathrm{O}_{2}$ by metal-catalysed histidine oxidation. Nature 2006, 440, 363-367. [CrossRef] [PubMed]

31. Jacquamet, L.; Traore, D.A.; Ferrer, J.L.; Proux, O.; Testemale, D.; Hazemann, J.L.; Nazarenko, E.; El Ghazouani, A.; Caux-Thang, C.; Duarte, V.; et al. Structural characterization of the active form of PerR: Insights into the metal-induced activation of PerR and Fur proteins for DNA binding. Mol. Microbiol. 2009, 73, 20-31. [CrossRef]

32. Lund, E.K.; Wharf, S.G.; Fairweather-Tait, S.J.; Johnson, I.T. Increases in the concentrations of available iron in response to dietary iron supplementation are associated with changes in crypt cell proliferation in rat large intestine. J. Nutr. 1998, 128, 175-179. [CrossRef] [PubMed]

33. Naikare, H.; Palyada, K.; Panciera, R.; Marlow, D.; Stintzi, A. Major role for FeoB in Campylobacter jejuni ferrous iron acquisition, gut colonization, and intracellular survival. Infect. Immun. 2006, 74, 5433-5444. [CrossRef] [PubMed]

34. Miller, C.E.; Rock, J.D.; Ridley, K.A.; Williams, P.H.; Ketley, J.M. Utilization of lactoferrin-bound and transferrin-bound iron by Campylobacter jejuni. J. Bacteriol. 2008, 190, 1900-1911. [CrossRef] [PubMed]

35. Xu, F.; Zeng, X.; Haigh, R.D.; Ketley, J.M.; Lin, J. Identification and characterization of a new ferric enterobactin receptor, CfrB, in Campylobacter. J. Bacteriol. 2010, 192, 4425-4435. [CrossRef] 